Highly Energetic Physical Processes and

Mechanisms for Emission from Astrophysical Plasmas

IAU Symposium, Vol. 195, 2000

P. C. H. Martens, S. Tsuruta, and M. A. Weber, eds.

\title{
Electron Trapping and Precipitation in Asymmetric Solar Flare Loops
}

\author{
M. J. Aschwanden and L. Fletcher \\ Lockheed Martin Advanced Technology Center, Solar and Astrophysics \\ Lab., Dept. L9-41, Bldg. 252, 3251 Hanover St., Palo Alto, CA 94304, \\ U.S.A.
}

T. Sakao

Natl. Astronomical Observatory, 2-21-1 Osawa, Mitaka, Tokyo 181-8599, Japan

T. Kosugi

Inst. Space and Astronautical Science, 3-1-1 Yoshinodai, Sagamihara, Kanagawa 229-8510, Japan

H. Hudson

Solar Physics Research Corporation, 4720 Calle Desecada, Tucson, AZ 85718, U.S.A.

\begin{abstract}
Acceleration, propagation, and energy loss of particles energized in solar flares cannot be studied separately because their radiative signatures observed in the form of hard X-ray bremsstrahlung or radio gyrosynchrotron emission represent a convolution of all these processes. We analyze hard X-ray emission from solar flares using a kinematic model that includes free-streaming electrons (having an energy-dependent timeof-flight delay) as well as temporarily trapped electrons (which are pitchangle scattered by Coulomb collisional scattering) to determine various physical parameters (trapping times, flux asymmetry, loss-cone angles, magnetic mirror ratios) in flare loops with asymmetric magnetic fields.
\end{abstract}

The theoretical model of deconvolving directly-precipitating and trap-precipitating electrons from solar-flare hard X-ray time profiles is described in Aschwanden (1998).

We analyze the footpoint separation $d$ and flux asymmetry $A$ of magnetically conjugate double footpoint sources in hard X-ray images from the Yohkoh Hard X-Ray Telescope (HXT). The dataset of 54 solar flares includes all events simultaneously observed with the Compton Gamma Ray Observatory (CGRO) in high time-resolution mode. From the CGRO data, we had previously deconvolved the direct-precipitation and trap-precipitation components (Aschwanden, Schwartz, \& Dennis 1998).

Using the combined measurements from CGRO and HXT, we develop an asymmetric trap model that allows us to quantify the relative fractions of four different electron components, i.e., the ratios of direct-precipitating $\left(q_{P 1}, q_{P 2}\right)$ 

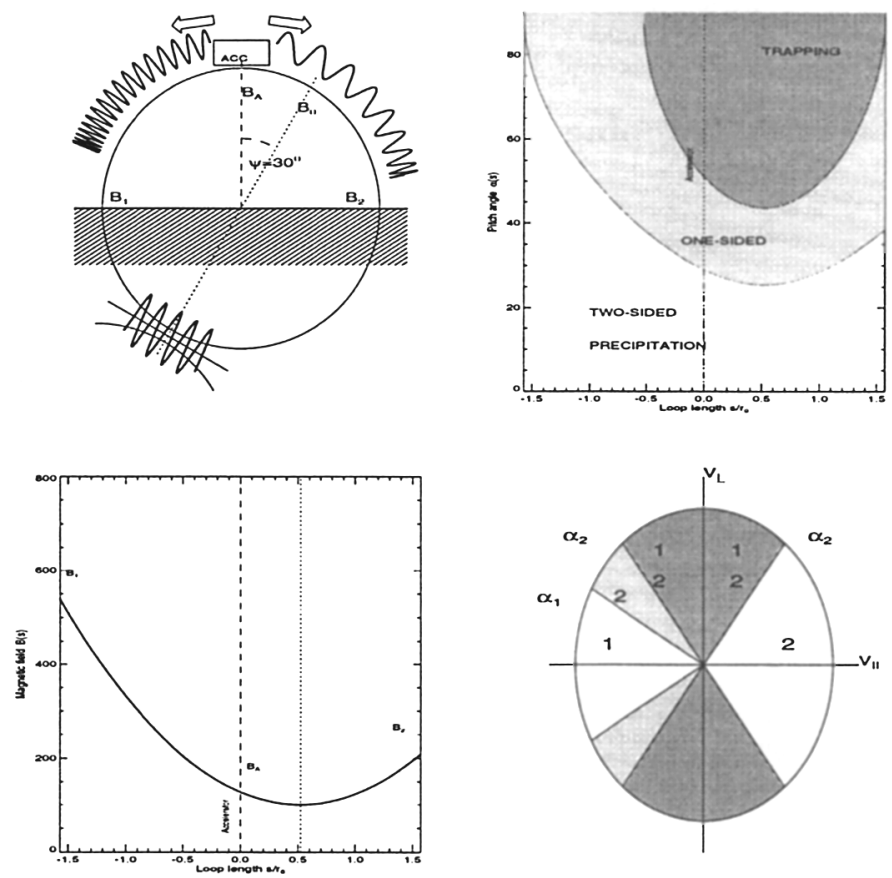

Figure 1. Geometry (left top), magnetic field strength (left bottom), pitch angle variation (right top), and velocity distribution (right bottom) of trapped particles in an asymmetric trap.

and trap-precipitating electrons $\left(q_{T 1}, q_{T 2}\right)$ at both magnetically conjugate footpoints (Aschwanden et al. 1999). We find mean ratios of $q_{P 1}=0.14 \pm 0.06$, $q_{P 2}=0.26 \pm 0.10$, and $q_{T}=q_{T 1}+q_{T 2}=0.60 \pm 0.13$. We assume an isotropic pitchangle distribution at the acceleration site and double-sided trap precipitation $\left(q_{T 2} / q_{T 1}=q_{P 2} / q_{P 1}\right)$ to determine the conjugate loss-cone angles $\left(\alpha_{1}=42^{0} \pm 11^{0}\right.$ and $\left.\alpha_{2}=52^{0} \pm 10^{0}\right)$ and magnetic mirror ratios at both footpoints $\left(R_{1}=1.6-4.0\right.$ and $R_{2}=1.3-2.5$ ).

From the relative displacement of footpoint sources, we also measure altitude differences of HXR emission at different energies, which are found to decrease systematically with higher energies, with a statistical height difference of $h_{L o}-h_{M 1}=980 \pm 250 \mathrm{~km}$ and $h_{M 1}-h_{M 2}=310 \pm 300 \mathrm{~km}$ between the 3 lower HXT energy channels (L0, M1, M2).

\section{References}

Aschwanden, M. J. 1998, ApJ, 502, 455

Aschwanden, M. J., Schwartz, R. A., \& Dennis, B. R. 1998, ApJ, 502, 468

Aschwanden, M. J., Fletcher, L., Sakao, T., Kosugi, T., \& Hudson, H. 1999, ApJ, 517, 977 